\title{
Depletion interactions of non-spherical colloidal particles in polymer solutions
}

\author{
E. Eisenriegler and A. Bringer \\ Institut für Festkörperforschung, \\ Forschungszentrum Jülich, \\ D-52425 Jülich, Germany
}

(Dated: November 15, 2018)

\begin{abstract}
We consider anisotropic colloidal particles immersed in a solution of long, flexible, and nonadsorbing polymers. For the dumbbell shapes of recently synthesized particles consisting of two intersecting spheres and for lens-shaped particles with spherical surfaces we calculate the isotropic and anisotropic interaction parameters that determine the immersion free energy and the orientationdependent depletion interaction between particles that are induced by the polymers. Exact results are obtained for random-walk like (ideal) polymer chains.
\end{abstract}




\section{INTRODUCTION}

In colloidal suspensions containing polymer chains, there are tunable effective interactions between the colloid particles. Free nonadsorbing polymer chains avoid the space between two particles, leading to an unbalanced pressure, which pushes them towards each other. Such depletion forces for an isolated pair of immersed particles or for a single immersed particle near a wall have been measured in recent experiments $\frac{1}{1}$.

Here we consider anisotropic colloid particles. For the dumbbell shapes of recently synthesized $^{2}$ particles consisting of two intersecting spheres, and for lens-shaped particles with spherical surfaces, as in Fig. 1, we calculate the immersion free energy and the orientation-dependent depletion interaction. The predictions are compared with results for prolate and oblate ellipsoids ${ }^{3.4 .5}$, which have also a symmetry axis of revolution and a symmetry center of reflection.

The case of large particle to polymer size ratio can be investigated by means of small curvature expansions of the Helfrich or Derjaguin type, but here we consider mesoscopic particles which are small compared to characteristic polymer lengths such as the gyration radius $\mathcal{R}_{g}$, and we concentrate on the case of ideal, random-walk like, polymers. The well known correspondence ${ }^{6}$ between the statistics of long flexible polymers and critical field theories allows us to use the small particle operator-expansion ${ }^{7.8 .9}$ for predicting the polymerinduced interactions. The operator weights in the expansion for dumbbells and lenses are calculated by a conformal mapping to a wedge-geometry.

We introduce the polymer-magnet analogy and small particle expansion in Sec. II, discuss density-profiles in a wedge and outside a dumbbell or lens in Sec III, and evaluate, for ideal polymers, the corresponding small particle amplitudes in the Gaussian model in Sec IV. These results are compared with corresponding results for ellipsoids and, in Sec. V, with a more general class of weakly anisotropic particles. In Sec. VI the amplitudes are used to determine the orientation-dependent interactions, and in Sec VII we summarize the new results. Some technical details are relegated to Appendices A-D. 


\section{POLYMER-MAGNET ANALOGY AND SMALL PARTICLE EXPANSION}

In the polymer-magnet analogy the partition function of a polymer chain with ends at $\mathbf{r}_{1}$ and $\mathbf{r}_{2}$ corresponds to the order-parameter correlation function $\left\langle\varphi_{12}\right\rangle$ of a Ginzburg-Landau model or field theory ${ }^{5.6}$. Here $\varphi_{12}$ is the product $\Phi\left(\mathbf{r}_{1}\right) \Phi\left(\mathbf{r}_{2}\right)$ of two order parameter fields $\Phi$. Ideal polymers correspond to a Gaussian Ginzburg-Landau model with Hamiltonian

$$
H=\int d \mathbf{r}\left[\frac{1}{2}(\nabla \Phi)^{2}+\frac{t}{2} \Phi^{2}\right]
$$

where the integration extends over the volume outside the particles, and where the order parameter satisfies the Dirichlet boundary condition

$$
\Phi=0
$$

at the particle surfaces, since we consider nonadsorbing polymers. We always consider length scales much larger than the persistence and extrapolation lengths.

The free energy $F$ it costs to immerse particles in a dilute polymer solution in an unbounded space or in the half space bounded by a wall is determined by the polymer partition functions with and without the particles and is given by ${ }^{4.5}$

$$
F / p_{0}=-\mathcal{L} \int d \mathbf{r}_{1} d \mathbf{r}_{2}\left[\left\langle\varphi_{12}\right\rangle_{\mathcal{H}+\delta \mathcal{H}}-\left\langle\varphi_{12}\right\rangle_{\mathcal{H}}\right]
$$

Here $p_{0}=n k_{B} T$ is the ideal gas pressure in the dilute solution with chain density $n$, and $\mathcal{H}+$ $\delta \mathcal{H}$ and $\mathcal{H}$ denote Ginzburg-Landau Hamiltonians of the form (2.1) in presence and absence of the particles, respectively. The dependence of the double integral on the temperature

deviation $t$ from the critical point is converted into the dependence on $\mathcal{R}_{g}^{2}$ of $-F / p_{0}$ by means of the inverse Laplace transform $\mathcal{L}(.)=.\int(d t / 2 \pi i) \exp \left(3 t \mathcal{R}_{g}^{2} / d\right)(.$.$) where d$ denotes the spatial dimension.

Consider particles with a size much smaller than $\mathcal{R}_{g}$ and a shape that is symmetric about both a center of reflection and an axis of revolution. Examples are rods, disks, ellipsoids, dumbbells, and lenses. In the spirit of the operator-product expansion, a small mesoscopic perturbation in a critical field theory can be represented by a sum of point operators. Thus, for a single small particle $\mathrm{e}^{7,8,9,10}$ with center at $\mathbf{r}_{\mathrm{P}}$,

$$
e^{-\delta \mathcal{H}} \propto 1+\sigma_{I}+\sigma_{A},
$$


where

$$
\begin{gathered}
\sigma_{I}=a_{1} \epsilon\left(\mathbf{r}_{\mathrm{P}}\right)+\ldots \\
\sigma_{A}=b_{1} \partial_{\|}^{2} \epsilon\left(\mathbf{r}_{\mathrm{P}}\right)+b_{2} T_{\|\|}\left(\mathbf{r}_{\mathrm{P}}\right)+\ldots
\end{gathered}
$$

are linear combinations of isotropic(I) and anisotropic(A) operators from the operator algebra of the Ginzburg-Landau model, which reflect the symmetries of the particle shape and boundary condition. Here $\epsilon \propto-\Phi^{2}$ is the energy density, $\partial_{\|}$is a derivative along the particle axis, and $T_{\|||}$is the diagonal component of the stress tensor of the field theory along the axis.

Only the operators of lowest scaling dimensions $x=d-1 / \nu, d+2-1 / \nu, d$ are shown in Eqs. (2.4)-(2.6), and their coefficients $a_{1}, b_{1}, b_{2}$ by scale invariance must be proportional to the particle size raised to the power $x$. For ideal chains (Gaussian model) the Flory (correlation length) exponent $\nu$ equals $1 / 2$, so that $\partial_{\|}^{2} \epsilon$ and $T_{\|\mid\|}$have the same scaling dimension $d$, and the $b_{1}$ and $b_{2}$ terms both contribute to the leading anisotropic behavior. For chains with excluded volume interaction (corresponding ${ }^{6}$ to the $N$-vector model in the limit $N \rightarrow 0$ ), $\nu$ is larger than $1 / 2$, and we expect that the $b_{2}$ term dominates the anisotropic behavior of a small particle.

The coefficients $a_{1}, b_{1}, b_{2}$ depend on the size and shape of the particle but are independent of other distant particles, of the distant boundary wall of the half space, and ${ }^{11}$ of $t$. Thus we evaluate the coefficients for a single particle in an unbounded space at $t=0$, and then use them to make predictions for the interaction between particles or a particle and a wall. The coefficients can be evaluated from the density profiles $\langle\epsilon(\mathbf{r})\rangle$ and $\left\langle T_{k l}(\mathbf{r})\right\rangle$ of the energy density and the stress tensor that are induced by a single particle. While ellipsoids have been considered in Refs. 4, 5 we concentrate here on dumbbells and lens-shaped particles.

\section{DENSITIES IN A WEDGE AND OUTSIDE A DUMBBELL OR LENS}

A system at the critical point containing a colloidal dumbbell composed of two overlapping spheres, or a lens with two spherical surfaces, can be conformally mapped onto a critical system filling a wedge with opening angle $\alpha$, which is smaller or larger than $\pi$ in case of the dumbbell or lens, as in Fig. 1. As explained in more detail in Ref. 9, an inversion about 
the point denoted by the heavy dot on the left hand side of Fig. 1 maps the interior of the wedge onto the exterior of a particle with a dumbbell or lens shape. The two boundary half planes of the wedge and the edge where they meet are mapped onto the two spherical surfaces of the particle and the circle $C$ of diameter $\mathcal{D}$ where they intersect. $\mathcal{D}$ is related to the diameter $L$ of the two spheres by $\mathcal{D}=L \sin (\alpha / 2)$. For $\alpha=0, \pi$, and $2 \pi$ the particle on the right hand side of Fig. 1 becomes a dumbbell of two touching spheres, a spherical particle, and a circular disk, respectively.

For the wedge the boundary-induced density profile of a scalar operator $\mathcal{O}$, such as the energy density $\epsilon$, has the form $12,13.14$

$$
\left\langle\mathcal{O}\left(\hat{\mathbf{r}}_{\mathrm{e}}, \rho, \Omega\right)\right\rangle_{\text {wedge }}^{(i, j)}=B_{\mathcal{O}}^{1 / 2} \rho^{-x_{\mathcal{O}}} \overline{\mathcal{P}}_{i, j}(\alpha, \Omega)
$$

Here the position vector $\hat{\mathbf{r}}$ is expressed in cylindrical coordinates $\left(\hat{\mathbf{r}}_{\mathrm{e}}, \rho, \Omega\right)$, where the edge of the wedge is the axis. The component $\hat{\mathbf{r}}_{\mathrm{e}}$ is parallel to the edge (and in general has dimension $d-2$ ), and the two component vector perpendicular to the edge is determined by its angle $\Omega$ with the symmetry half plane of the wedge, i.e. $-\alpha / 2 \leq \Omega \leq \alpha / 2$, and by the distance $\rho$ from the edge. The indices $(i, j)$ characterize the surface universality classes $\frac{15}{}$ of the two boundary half planes $\Omega=(-\alpha / 2, \alpha / 2)$ of the wedge. While $\overline{\mathcal{P}}$ is a universal scaling function, $B_{\mathcal{O}}$ is the non-universal amplitude in the pair correlation function

$$
\langle\mathcal{O}(\mathbf{r}) \mathcal{O}(0)\rangle_{\text {bulk }}=B_{\mathcal{O}} r^{-2 x_{\mathcal{O}}}
$$

of $\mathcal{O}$ in the bulk.

The corresponding density $\langle\mathcal{O}(\mathbf{r})\rangle_{\text {particle }}$ outside a dumbbell or lens follows ${ }^{12}$ from (3.1) and the conformal mapping and has the form given in Eqs. (5.14) and (5.10) of Ref. 9. Turning to the energy density $\mathcal{O}=\epsilon$ with scaling function $\overline{\mathcal{P}}=\overline{\mathcal{E}}$ and equal boundaries $i=j$, the behavior

$$
\overline{\mathcal{E}}_{i, i}(\alpha, \Omega)=e_{0}^{(i)}(\alpha)\left[1+e_{2}^{(i)}(\alpha) \Omega^{2} / 2+\ldots\right]
$$

near the symmetry half plane $\Omega=0$ of the wedge determines the profile $\langle\epsilon(\mathbf{r})\rangle_{\text {particle far from }}$ the dumbbell or lens and yields 9

$$
a_{1}^{(i)}=\mathcal{D}^{x_{\epsilon}} e_{0}^{(i)}(\alpha) / B_{\epsilon}^{1 / 2}
$$

and

$$
b_{1}^{(i)}=a_{1}^{(i)} \frac{\mathcal{D}^{2}}{8 x_{\epsilon}\left(x_{\epsilon}+1\right)}\left(e_{2}^{(i)}(\alpha)-x_{\epsilon}\right)
$$


for two leading coefficients in the small particle expansion.

The boundary-induced density profile $\left\langle T_{\kappa \lambda}(\hat{\mathbf{r}})\right\rangle_{\text {wedge }}$ of the stress tensor in the wedge is given by

$$
\left\langle T_{\kappa \lambda}\left(\hat{\mathbf{r}}_{\mathrm{e}}, \rho, \Omega\right)\right\rangle_{\mathrm{wedge}}^{(i, j)}=\rho^{-d} \tau_{i, j}(\alpha)\left[\delta_{\kappa \lambda}-d u_{\kappa}^{(n)}(\Omega) u_{\lambda}^{(n)}(\Omega)\right]
$$

where $\mathbf{u}^{(n)}(\Omega)$ is the unit vector normal to the half plane $\Omega=$ const which contains $\hat{\mathbf{r}}$, as in the left hand side of Fig. 2. Unlike (3.1) there is no nonuniversal amplitude in (3.6), and the $\rho$-exponent and the $\Omega$-dependence are trivial. Only the variation of the universal amplitude $\tau$ with the opening angle $\alpha$ of the wedge depends on the bulk universality class and the surface classes $i, j$, and remains to be determined. The stress tensor density (3.6) has a vanishing trace and obeys the continuity equation, as discussed in Appendix A.

We note two special cases: (i) For $\alpha \rightarrow \pi$ and $i=j$ the wedge becomes the half space with a uniform boundary, and $\tau \rightarrow 0$ since the stress tensor density vanishes 12 . According to the left hand side of Fig. 2, only for $\tau=0$ is (3.6) consistent with the symmetries of the half space. (ii) For $\alpha \rightarrow 0, \tau$ diverges as

$$
\tau_{i, j} \rightarrow \alpha^{-d}\left(-\Delta_{i, j}\right)
$$

where $\Delta_{i, j}$ is the universal amplitude which determines the stress tensor density in the parallel plate geometry $\frac{12}{2}$. If the width of the film is $\omega$ and both tensor components are parallel to the plates, $\left\langle T_{\text {parallel,parallel }}\right\rangle_{\text {film }}^{(i, j)}=\omega^{-d}\left(-\Delta_{i, j}\right)$.

Using the inversion transformation for the conformal stress tensor ${ }^{9.16}$ one finds from Eq. (3.6) the stress tensor density

$$
\left\langle T_{k l}(\mathbf{r})\right\rangle_{\text {particle }}^{(i, j)}=\left(\mathcal{D} / \Lambda^{2}\right)^{d} \tau_{i, j}(\alpha)\left[\delta_{k l}-d u_{k}^{(N)}(\mathbf{r}) u_{l}^{(N)}(\mathbf{r})\right]
$$

outside the dumbbell or lens. Here

$$
\Lambda^{2}=\sqrt{\left[r^{2}-(\mathcal{D} / 2)^{2}\right]^{2}+\mathcal{D}^{2} r_{\|}^{2}}
$$

with $r$ and $r_{\|}$the distance of point $\mathbf{r}$ from the particle center and its component parallel to the particle rotation axis. The inverse length $\mathcal{D} / \Lambda^{2}$ in $(3.8)$ equals $b(\hat{\mathbf{r}}) / \rho(\hat{\mathbf{r}})$, where $b$ is the dilatation factor $|\operatorname{det}(\partial \hat{\mathbf{r}} / \partial \mathbf{r})|^{1 / d}$ of the conformal mapping, and diverges as $\left(r_{\|}, r\right) \rightarrow$ $(0, \mathcal{D} / 2)$, as the circle $C$ of intersection is approached. The unit vector $\mathbf{u}^{(N)}$ in the particle geometry is the counterpart of $\mathbf{u}^{(n)}$ in the wedge geometry. It points along the surface normal at $\mathbf{r}$ of the spherical surface portion $S_{C, \mathbf{r}}$ which contains $\mathbf{r}$ and is bounded by the circle $C$. 
$S_{C, \mathbf{r}}$ is the image of the half plane $\Omega=$ const which contains $\hat{\mathbf{r}}$. With the particle axis $\|$ as one of the Cartesian directions,

$$
u_{k}^{(N)}(\mathbf{r})=\Lambda^{-2}\left\{\delta_{k \|}\left[r^{2}-(\mathcal{D} / 2)^{2}\right]-2 r_{k} r_{\|}\right\} .
$$

The vector field $\mathbf{u}^{(N)}$ for given $\mathcal{D}$ is shown on the right hand side of Fig. 2. Both $\mathbf{u}^{(N)}$ and $\mathbf{u}^{(n)}$ are independent of $\alpha$ and the bulk and surface universality classes $i, j$.

In leading order $\mathcal{D} \ll r$,

$$
\left\langle T_{k l}(\mathbf{r})\right\rangle_{\text {particle }}^{(i, j)} \rightarrow\left(\mathcal{D} / r^{2}\right)^{d} \tau_{i, j}(\alpha)\left[\delta_{k l}-d \mathcal{I}_{k, \|} \mathcal{I}_{l, \|}\right],
$$

with

$$
\mathcal{I}_{k, m} \equiv \mathcal{I}_{k, m}(\mathbf{r})=\delta_{k, m}-2 r_{k} r_{m} / r^{2} .
$$

On comparing with the stress tensor correlation function

$$
\left\langle T_{k l}(\mathbf{r}) T_{m n}(0)\right\rangle_{\mathrm{bulk}}=B_{T} r^{-2 d}\left\{(1 / 2)\left[\mathcal{I}_{k, m} \mathcal{I}_{l, n}+\mathcal{I}_{k, n} \mathcal{I}_{l, m}\right]-(1 / d) \delta_{k l} \delta_{m n}\right\}
$$

in unbounded bulk ${ }^{9.16 .17}$, Eq. (3.11) implies that

$$
\left\langle T_{k l}(\mathbf{r})\right\rangle_{\text {particle }}^{(i, j)} \rightarrow b_{2}^{(i, j)}\left\langle T_{k l}(\mathbf{r}) T_{\|\|}(0)\right\rangle_{\text {bulk }} \quad, \quad \mathcal{D} \ll r
$$

with the stress tensor contribution in the small particle expansion given by

$$
b_{2}^{(i, j)} T_{\|\|}=-\mathcal{D}^{d} \tau_{i, j}(\alpha) \frac{d}{B_{T}} T_{\|\|} .
$$

For the special case $\alpha \rightarrow 0$, where $\mathcal{D} \rightarrow \alpha L / 2$ and Eq. (3.7) applies, Eq. (3.15) reduces to the expression $b_{2}^{(i, j)} \rightarrow(L / 2)^{d} \Delta_{i, j} d / B_{T}$ for a dumbbell of two touching spheres with diameter $L$, given in Eq. (2.15) of Ref. 9 .

The form of $\langle T\rangle_{\text {wedge }}^{(i, j)}$ in (3.6) has been calculated for special cases. See Refs. 12,18 for $d=2$, Ref. 14 for symmetry breaking surfaces $i=j$ as $d \rightarrow 4$, and Appendix B for Dirichlet boundaries and $\alpha=\pi / 2$. Here we indicate how (3.6) can be derived in the general case. For $\mathbf{r}$ on the particle rotation axis, $\langle T\rangle_{\text {particle }}^{(i, j)}$ must have an eigenvector parallel to the axis, by rotation symmetry. That the particle axis is the image of a circle in the plane $\hat{\mathbf{r}}_{\mathrm{e}}=0$ of the wedge with center in the edge and passing through the center of inversion, see the long dashes in Figs. 1 and 2, implies an eigenvector $\mathbf{u}^{(n)}$ of $\langle T\rangle_{\text {wedge }}^{(i, j)}$ tangent to this circle. Likewise, degenerate eigenvectors perpendicular to the axis imply $d-1$ degenerate 
eigenvectors perpendicular to $\mathbf{u}^{(n)}$. The simple form (3.6) of the symmetric, traceless, and conformal tensor density $\langle T\rangle$ for the wedge then follows from scaling (dilatation symmetry), translation, and reflection symmetry in the edge-subspace, and the $\Omega$-independence of $\tau$ is due to the continuity equation of the stress tensor, see Appendix A. In Appendix B we also discuss, within the Gaussian model, the more complicated form of the density $\left\langle T^{(\text {can })}\right\rangle_{\text {wedge }}$ of the canonical stress tensor, which also obeys the continuity equation, but is not trace-free and not a conformal tensor, and for which $\mathbf{u}^{(n)}(\Omega)$, in general, is not an eigenvector. Its eigenvalues depend on both $\rho$ and $\Omega$, and some of the eigenvalues diverge as the boundary planes of the wedge are approached. The simple form (3.6) is recovered on adding the 'improvement-term'19.

We briefly comment on particle shapes where the horizontal axis on the right hand side of Fig. 1 (passing through the small triangle and square) is the rotation axis. These particles resemble an apple (self-intersecting torus) for $\alpha<\pi$ and an american football for $\alpha>\pi$ and could be conformally mapped onto a cone with opening angle $\alpha$.

\section{GAUSSIAN MODEL WITH DIRICHLET BOUNDARIES}

In $d=2$ spatial dimensions, a wedge can be obtained from the half plane by means of an appropriate conformal transformation. The dependence on the angle $\alpha$ only enters via the transformation and is to a large extent model-independent. For example, for $i=j$, the stress-amplitude $\tau$ is independent of the surface universality class $i$ and equals $\left[(\pi / \alpha)^{2}-\right.$ $1] c /(24 \pi)$, where only the universal bulk-constant $c$, the 'conformal charge', depends on the model class. Using (3.7), this is consistent with the $i$-independent film-amplitude $\Delta_{i, i}=$ $-\pi c / 24$ for a strip with equal boundary conditions ${ }^{12}$. Also the form of the scaling functions $\overline{\mathcal{P}}_{i, i}=\mathcal{A}_{\mathcal{O}}^{(i)}[(\alpha / \pi) \cos (\pi \Omega / \alpha)]^{-x_{\mathcal{O}}}$ is to a large extent model-independent ${ }^{13}$ and is completely determined by the bulk scaling index $x_{\mathcal{O}}$ and the universal, but $i$-dependent, half-space boundary amplitude $\mathcal{A}_{\mathcal{O}}^{(i)}$ of the scalar operator $\mathcal{O}$. For $i$ not equal to $j$, the stress amplitude $\tau_{i, j}=-(c /(24 \pi))-\Delta_{i, j} / \alpha^{2}$ also depends on the boundary universality classes. Since $\Delta_{i, j}$ for $i \neq j$ is in general different from $-\pi c / 24, \tau_{i, j}$ is nonvanishing even for $\alpha=\pi$, i.e. for the half plane with a non-homogeneous boundary ${ }^{18}$.

In $d>2$, however, no such conformal transformation exists, and the $\alpha$-dependent quantities $\overline{\mathcal{P}}$ and $\tau$ depend on the bulk and surface universality classes in a much stronger way. In 
particular a bulk amplitude (like $c$ ) and the parallel plate amplitude $\Delta_{i, j}$, are not sufficient to predict $\tau_{i, j}(\alpha)$.

For the Gaussian Ginzburg-Landau field theory (2.1) at the critical point $t=0$ in $d>2$ spatial dimensions, the scaling function $\overline{\mathcal{E}}$ of the energy density in the wedge is given by

$$
\overline{\mathcal{E}}=-\left\langle\Phi^{2}(\hat{\mathbf{r}})\right\rangle_{\text {wedge }} \rho^{d-2} /\left(\sqrt{2} \tilde{S}_{d}\right) \quad, \quad \tilde{S}_{d}=\pi^{-d / 2} \Gamma((d / 2)-1) / 4
$$

where we have suppressed the indices $(i, j)=(\mathrm{D}, \mathrm{D})$ with $\mathrm{D}$ for Dirichlet ${ }^{15}$. The stress tensor in the Gaussian model is the sum¹9

$$
T_{\kappa \lambda}(\hat{\mathbf{r}})=T_{\kappa \lambda}^{(\mathrm{can})}(\hat{\mathbf{r}})-I_{\kappa \lambda}(\hat{\mathbf{r}})
$$

of the canonical stress tensor

$$
T_{\kappa \lambda}^{(\text {can })}(\hat{\mathbf{r}})=\left(\hat{\partial}_{\kappa} \Phi(\hat{\mathbf{r}})\right)\left(\hat{\partial}_{\lambda} \Phi(\hat{\mathbf{r}})\right)-\delta_{\kappa \lambda}(\hat{\nabla} \Phi(\hat{\mathbf{r}}))^{2} / 2
$$

and the so-called improvement term with

$$
I_{\kappa \lambda}(\hat{\mathbf{r}})=\frac{1}{4} \frac{d-2}{d-1}\left[\hat{\partial}_{\kappa} \hat{\partial}_{\lambda}-\delta_{\kappa \lambda} \hat{\Delta}\right] \Phi^{2}(\hat{\mathbf{r}})
$$

Unlike $T^{(\mathrm{can})}$, the density of $T$ in (4.2) has a vanishing trace and transforms as a conformal tensor, see Appendices B and C. The bulk amplitude $B_{T}$ in (3.13) equals $\left[\tilde{S}_{d}(d-2)\right]^{2} d /(d-1)$ for the Gaussian model.

The boundary-induced densities $\left\langle\Phi^{2}(\hat{\mathbf{r}})\right\rangle_{\text {wedge }}$ and $\left\langle T_{\kappa \lambda}(\hat{\mathbf{r}})\right\rangle_{\text {wedge }}$ follow in an obvious way from the boundary-induced contribution $\delta\left\langle\hat{\varphi}_{12}\right\rangle_{\text {wedge }}$ of the propagator in the wedge

$$
\left\langle\hat{\varphi}_{12}\right\rangle_{\text {wedge }}=\left\langle\hat{\varphi}_{12}\right\rangle_{\text {bulk }}+\delta\left\langle\hat{\varphi}_{12}\right\rangle_{\text {wedge }}
$$

with

$$
\hat{\varphi}_{12}=\Phi\left(\hat{\mathbf{r}}_{1}\right) \Phi\left(\hat{\mathbf{r}}_{2}\right) .
$$

For the special opening angles $\alpha=\pi / g$, with $g$ a positive integer, $\delta\left\langle\hat{\varphi}_{12}\right\rangle_{\text {wedge }}$ is a linear combination of $2 g-1$ bulk propagators from $\mathbf{r}_{1}$ to images of $\mathbf{r}_{2}$, see Appendix B.

In order to determine $e_{0}, e_{2}$ in (3.3) and $\tau$ in (3.6) for arbitrary $\alpha$, we use the representation of Ref. 20 at the critical point

$$
\begin{aligned}
\left\langle\hat{\varphi}_{12}\right\rangle_{\text {wedge }}=\int d^{d-2} q(2 \pi)^{2-d} & e^{i \mathbf{q}\left(\hat{\mathbf{r}}_{e 1}-\hat{\mathbf{r}}_{e 2}\right)}(2 / \alpha) \sum_{m=1}^{\infty} I_{m \pi / \alpha}\left(q \rho_{<}\right) K_{m \pi / \alpha}\left(q \rho_{>}\right) \\
& \times \sin \left[m \pi\left(\frac{1}{2}+\frac{\Omega_{1}}{\alpha}\right)\right] \sin \left[m \pi\left(\frac{1}{2}+\frac{\Omega_{2}}{\alpha}\right)\right],
\end{aligned}
$$


where $\rho_{<}=\min \left(\rho_{1}, \rho_{2}\right), \rho_{>}=\max \left(\rho_{1}, \rho_{2}\right)$, and $I$ and $K$ are modified Bessel functions. A more explicit expression arises for $\left(\hat{\mathbf{r}}_{\mathrm{e} 1}, \rho_{1}\right)=\left(\hat{\mathbf{r}}_{\mathrm{e} 2}, \rho_{2}\right)$. Using

$$
\int_{0}^{\infty} d x x^{2 A-1} I_{m \pi / \alpha}(x) K_{m \pi / \alpha}(x)=2^{2 A-2} \frac{\Gamma(A)}{\Gamma(1-A)} \int_{0}^{1} d t t^{m \pi / \alpha} t^{A-1}(1-t)^{-2 A}
$$

for $2 A=d-2$ in order to rewrite the $q$-integral in a form where the $m$-summation can be done $e^{20}$, one finds

$$
\begin{aligned}
& \left.\tilde{S}_{d}^{-1}\left\langle\hat{\varphi}_{12}\right\rangle_{\text {wedge }}\right|_{\left(\hat{\mathbf{r}}_{\mathrm{e} 1}, \rho_{1}\right)=\left(\hat{\mathbf{r}}_{\mathrm{e} 2}, \rho_{2}\right)}=-(2 / \alpha) \sin (\pi d / 2) \rho^{2-d} \int_{0}^{1} d t \Psi_{d}(t) \\
& \times\left\{\frac{1-t^{\pi / \alpha} \cos \left[\left(\Omega_{1}-\Omega_{2}\right) \pi / \alpha\right]}{1+t^{2 \pi / \alpha}-2 t^{\pi / \alpha} \cos \left[\left(\Omega_{1}-\Omega_{2}\right) \pi / \alpha\right]}-\frac{1+t^{\pi / \alpha} \cos \left[\left(\Omega_{1}+\Omega_{2}\right) \pi / \alpha\right]}{1+t^{2 \pi / \alpha}+2 t^{\pi / \alpha} \cos \left[\left(\Omega_{1}+\Omega_{2}\right) \pi / \alpha\right]}\right\},
\end{aligned}
$$

where $\rho \equiv \rho_{1}=\rho_{2}$ and

$$
\Psi_{d}(t)=t^{(d-4) / 2}(1-t)^{2-d}
$$

The expression in Eq. (4.9) satisfies the Dirichlet boundary conditions since the curly bracket vanishes for $\Omega_{1}$ (or $\Omega_{2}$ ) equal to $\alpha / 2$ or $-\alpha / 2$. For $\Omega_{1} \neq \Omega_{2}$ the $t$-integral converges in the interval $2-(2 \pi / \alpha)<d<4$ of spatial dimensions $d$ with the lower and upper limits coming from the behaviors of the integrand for $t \searrow 0$ and $t \nearrow 1$, respectively. The bulk divergence $\left(\rho\left|\Omega_{1}-\Omega_{2}\right|\right)^{2-d}$ for $\left|\Omega_{1}-\Omega_{2}\right| \rightarrow 0$ and $d>2$ contained in (4.9) comes from integrating the first term in curly brackets over a region near $t=1$ where $1-t$ is of the order of $\left|\Omega_{1}-\Omega_{2}\right|$.

A convenient way to evaluate the boundary-induced density

$$
\left\langle\Phi^{2}(\hat{\mathbf{r}})\right\rangle_{\text {wedge }}=\lim _{\hat{\mathbf{r}}_{1} \rightarrow \hat{\mathbf{r}}, \hat{\mathbf{r}}_{2} \rightarrow \hat{\mathbf{r}}} \delta\left\langle\hat{\varphi}_{12}\right\rangle_{\text {wedge }}
$$

and its scaling function $\overline{\mathcal{E}}(\alpha, \Omega)$ in (4.1) for $d=3$ by means of Eq. (4.9), is based on the observation $^{21}$ that $\overline{\mathcal{E}}$ is an analytic function of $d$ in an interval that includes both $d=2$ and $d=3$ as interior points. Since the limit $\hat{\mathbf{r}}_{1} \rightarrow \hat{\mathbf{r}}, \hat{\mathbf{r}}_{2} \rightarrow \hat{\mathbf{r}}$ of the bulk propagator $\propto\left|\hat{\mathbf{r}}_{1}-\hat{\mathbf{r}}_{2}\right|^{2-d}$ vanishes for $d<2$ (while it is infinite for $d>2$ ), one may replace $\delta\left\langle\hat{\varphi}_{12}\right\rangle_{\text {wedge }}$ by $\left\langle\hat{\varphi}_{12}\right\rangle_{\text {wedge }}$ and use (4.9), in calculating the limit in (4.11) for $d<2$. This leads to

$$
\begin{aligned}
\overline{\mathcal{E}}(\alpha, \Omega) & =(\sqrt{2} / \alpha) \sin (\pi d / 2) \int_{0}^{1} d t \Psi_{d}(t) \\
& \times\left\{\frac{1}{1-t^{\pi / \alpha}}-\frac{1+t^{\pi / \alpha} \cos (2 \pi \Omega / \alpha)}{1+t^{2 \pi / \alpha}+2 t^{\pi / \alpha} \cos (2 \pi \Omega / \alpha)}\right\} \quad ; \quad d<2,
\end{aligned}
$$

in terms of an integral which is well defined for $2-(2 \pi / \alpha)<d<2$ and which has to be analytically continued ${ }^{21}$ in order to obtain $\overline{\mathcal{E}}$ for $d=3$. 
For $e_{0}(\alpha)=\overline{\mathcal{E}}(\alpha, \Omega=0)$ the integral in (4.12) becomes

$$
J(\alpha, d)=\int_{0}^{1} d t \Psi_{d}(t) t^{\pi / \alpha} \frac{2}{1-t^{2 \pi / \alpha}},
$$

and the continuation can be made by rewriting $J$ as the sum of $J^{(1)}$ and $J^{(2)}$, where

$$
J^{(1)}(\alpha, d)=\int_{0}^{1} d t \Psi_{d}(t) t^{\pi / \alpha}\left[\frac{2}{1-t^{2 \pi / \alpha}}-l(t)\right],
$$

and

$$
J^{(2)}(\alpha, d)=\int_{0}^{1} d t \Psi_{d}(t) t^{\pi / \alpha} l(t)
$$

Here

$$
l(t)=\frac{\alpha / \pi}{1-t}+1-\frac{\alpha}{2 \pi}
$$

are the first two terms in the Laurent series of $2 /\left(1-t^{2 \pi / \alpha}\right)$ around $t=1$ so that the integrability domain $2-(2 \pi / \alpha)<d<4$ of $J^{(1)}$ extends up to $d=4$. As a sum of betafunctions the continuation to $d=3$ of the integral $J^{(2)}$ is trivial and yields $J^{(2)}(\alpha, 3)=-1$, implying

$$
e_{0}(\alpha)=(\sqrt{2} / \alpha)\left[1-J^{(1)}(\alpha, 3)\right] \quad, \quad d=3
$$

Here $J^{(1)}(\alpha, 3)$ follows from the right hand side in Eq. (4.14) on replacing $\Psi_{d}$ by $\Psi_{3}=$ $t^{-1 / 2} /(1-t)$, and for $\alpha$ arbitrary between 0 and $2 \pi$ we have calculated it numerically. Using Eq. (3.4), the corresponding results for $a_{1} B_{\epsilon}^{1 / 2} / L=\sin (\alpha / 2) e_{0}(\alpha)$ in the interval $0<\alpha<\pi$ and $a_{1} B_{\epsilon}^{1 / 2} / \mathcal{D}=e_{0}(\alpha)$ in the interval $\pi<\alpha<2 \pi$ are shown in Fig. 3. Analytical results for some special values of $\alpha$ are given in Table 1 .

In the Gaussian model some amplitudes of isotropic operators beyond leading order in (2.5), such as $\Phi^{4}$ and $\Phi^{6}$, are also determined 22 by $a_{1}$.

To calculate the small particle anisotropy-amplitude $b_{1}$ in (3.5), we need the coefficient $\propto \Omega^{2}$ of $\overline{\mathcal{E}}$. The contribution of order $\Omega^{2}$ to the curly bracket in (4.12) contains a factor $1-t^{\pi / \alpha}$ and leads to a convergent integral up to $d=4$. Thus, no continuation is necessary, and

$$
e_{0}(\alpha) e_{2}(\alpha)=\left(4 \sqrt{2} \pi^{2} / \alpha^{3}\right) \int_{0}^{1} d t \Psi_{3}(t) t^{\pi / \alpha}\left(1-t^{\pi / \alpha}\right) /\left(1+t^{\pi / \alpha}\right)^{3} \quad, \quad d=3,
$$

which on using (3.5) with (3.4), (4.17) leads to the results for $b_{1}$ shown in Fig. 4 and in Table 1. 
In Appendix D we use similar continuations in $d$ to calculate the stress tensor amplitude $\tau(\alpha)$ from (3.6) and (4.2)-(4.4) in $d=3$ with the result (D15). The second anisotropyamplitude $b_{2}$ then follows from (3.15) and the value $d / B_{T}=32 \pi^{2}$, with the results shown in Fig. 5 and Table 1.

It is interesting to compare dumbbells and lenses with ellipsoids. We compare a dumbbell with $\alpha$ between 0 and $\pi$ with a prolate ellipsoid that circumscribes the dumbbell, touches it at the highest and lowest points, and has the same curvature at these points. Denoting by $D_{\|}$and $D_{\perp}$ the diameters of the ellipsoid parallel and perpendicular to the rotation axis,

$$
D_{\|}=2 L \cos ^{2}(\alpha / 4) \quad, \quad D_{\perp}=\sqrt{2} L \cos (\alpha / 4) .
$$

Similarly, we compare a lens with $\alpha$ between $\pi$ and $2 \pi$ with an oblate circumscribing ellipsoid, so that

$$
D_{\|}=\mathcal{D} \operatorname{ctg}(\alpha / 4) \quad, \quad D_{\perp}=\mathcal{D}
$$

where $\operatorname{D} \operatorname{ctg}(\alpha / 4)$ is the width ${ }^{9}$ of the lens. The amplitudes $a_{1}, b_{1}, b_{2}$ of the ellipsoids are shown as circles in Figs. 3-5. They follow from Refs. 11,23 and Eqs. (180)-(185) in Ref. 5 where the long axis $\left[D_{\|}, D_{\perp}\right]$ and short axis $\left[D_{\perp}, D_{\|}\right]$of the [prolate, oblate] ellipsoid is denoted by $l$ and $s$, respectively.

As expected, the isotropic and anisotropic perturbations of the polymer system from dumbbells are weaker and stronger, respectively, than from the circumscribing prolate ellipsoids. The oblate ellipsoids have stronger isotropic-perturbation amplitudes $a_{1}$ and also slightly stronger anisotropic amplitudes $b_{1}$ and $b_{2}$ than the lens.

\section{WEAK ANISOTROPY}

Consider the amplitudes $a_{1}, b_{1}$, and $b_{2}$ for particles with a surface $S^{\prime}$ which deviates only slightly from the surface $S$ of a sphere with radius $R . S^{\prime}$ is obtained by shifting each surface point $\mathbf{r}_{S}$ of $S$ by a small amount $\eta\left(\theta_{S}\right)$ toward the center of $S$ at the origin. Here $\theta_{S}$ is the angle which $\mathbf{r}_{S}$ encloses with the particle rotation axis, and we consider particles with a center of reflection so that $\eta\left(\theta_{S}\right)=\eta\left(\pi-\theta_{S}\right)$. For a dumbbell or lens with $\alpha=\pi+\delta \alpha$, we choose $R=L / 2$ and obtain

$$
\eta=\delta \alpha(L / 4)\left|\cos \theta_{S}\right|
$$


to first order in $\delta \alpha$. As expected from Fig. 1, $\eta$ in (5.1) is non-analytic at $\theta_{S}=\pi / 2$. At the end of Sec. III we have introduced apple- and football-shaped particles with $\alpha=\pi+\delta \alpha$ smaller and larger than $\pi$, respectively. Nearly spherical particles of this family are generated by

$$
\eta=\delta \alpha(L / 4) \sin \theta_{S}
$$

For weakly anisotropic prolate and oblate ellipsoids with $D_{\|}>D_{\perp}$ and $D_{\|}<D_{\perp}$, respectively,

$$
\eta=\frac{D_{\|}-D_{\perp}}{2} \sin ^{2} \theta_{S}
$$

if we choose $R=D_{\|} / 2$.

In the presence of the weakly anisotropic particle the propagator is given by ${ }^{\underline{5}}$

$$
\left\langle\varphi_{12}\right\rangle=\left\langle\varphi_{12}\right\rangle_{\text {sphere }}+\int d S \eta\left(\theta_{S}\right)\left\langle T_{\perp \perp}\left(\mathbf{r}_{S}\right) \varphi_{12}\right\rangle_{\text {sphere }}
$$

to first order in $\eta$. Here $\int d S$ is an integral over the surface $S$ of the sphere, and $T_{\perp \perp}$ is the diagonal component of the stress tensor perpendicular to $S$. Due to the Dirichlet boundary condition and the form (4.2)-(4.4) of the stress tensor, $T_{\perp \perp}\left(\mathbf{r}_{S}\right)$ can be replaced by $\left(\partial_{\perp} \Phi\right)^{2} / 2$ in the correlation function in (5.4) with the result

$$
\left\langle T_{\perp \perp}\left(\mathbf{r}_{S}\right) \varphi_{12}\right\rangle_{\text {sphere }}=\left[(d-2) \tilde{S}_{d}\right]^{2} \frac{\left(r_{1}^{2}-R^{2}\right)\left(r_{2}^{2}-R^{2}\right)}{R^{2}\left(\left|r_{1}-\mathbf{r}_{S}\right|\left|r_{2}-\mathbf{r}_{S}\right|\right)^{d}},
$$

and, from the behavior of (5.4) for $R \ll r_{1}, r_{2}$, one finds 24

$$
\begin{gathered}
a_{1} \sqrt{B_{\epsilon}}-R^{d-2} / \sqrt{2}=-\frac{(d-2) \Gamma(d / 2)}{\sqrt{2 \pi} \Gamma((d-1) / 2)} R^{d-3} \int_{0}^{\pi} d \theta_{S}\left(\sin \theta_{S}\right)^{d-2} \eta\left(\theta_{S}\right), \\
b_{1} \sqrt{B_{\epsilon}} / b_{2}=-\frac{1}{4 \sqrt{2} \pi^{d / 2}} \frac{d+1}{d-1} \Gamma((d+2) / 2),
\end{gathered}
$$

and

$$
b_{2}=R^{d-1} \frac{2 \pi^{(d-1) / 2}}{\Gamma((d+1) / 2)} \int_{0}^{\pi} d \theta_{S}\left(\sin \theta_{S}\right)^{d-2}\left[d\left(\cos \theta_{S}\right)^{2}-1\right] \eta\left(\theta_{S}\right) .
$$

Explicit expressions for dumbbells or lenses, apples or footballs, and ellipsoids in an arbitrary spatial dimension $d$ follow on inserting $\eta$ from (5.1)-(5.3). In $d=3$,

$$
\begin{aligned}
& a_{1} \sqrt{B_{\epsilon}} / L=\frac{1}{2 \sqrt{2}}-\delta \alpha\left(\frac{1}{8 \sqrt{2}}, \frac{\pi}{16 \sqrt{2}}\right), \\
& b_{2} / L^{3}=\delta \alpha\left(\frac{\pi}{16},-\frac{\pi^{2}}{64}\right)
\end{aligned}
$$


for (dumbbell or lens, apple or football), and

$$
\begin{aligned}
& a_{1} \sqrt{B_{\epsilon}}=\frac{D_{\|}}{2 \sqrt{2}}+\frac{D_{\perp}-D_{\|}}{3 \sqrt{2}}, \\
& b_{2}=\left(D_{\perp}-D_{\|}\right) D^{2} \frac{2 \pi}{15}
\end{aligned}
$$

for ellipsoids. In our first order calculation $D$ may be either $D_{\|}$or $D_{\perp}$. Note that $b_{2}$ is negative for the prolate shapes (dumbbell with $\delta \alpha<0$, football with $\delta \alpha>0$, and prolate ellipsoid with $D_{\|}>D_{\perp}$ ) and positive for the oblate shapes (lens with $\delta \alpha>0$, apple with $\delta \alpha<0$, and oblate ellipsoid with $\left.D_{\|}<D_{\perp}\right)$.

The amplitudes $b_{1}$ follow from the amplitudes $b_{2}$ above via the ratio

$$
b_{1} \sqrt{B_{\epsilon}} / b_{2}=-\frac{3}{8 \pi \sqrt{2}} .
$$

For a nearly spherical particle with rotation axis and reflection symmetry, the ratio of the anisotropy amplitudes $b_{1}$ and $b_{2}$ is independent of its shape, see (15.7) and (5.11), but the ratio becomes shape-dependent for larger deviations from spherical. For example,

$$
b_{1} \sqrt{B_{\epsilon}} / b_{2}=-\frac{1}{\pi}\left(\frac{7}{16 \sqrt{2}}, \frac{8+\sqrt{2}}{32}, \frac{5}{16 \sqrt{2}}, \frac{\ln \left(2 D_{\|} / D_{\perp}\right)}{4 \sqrt{2}}\right)
$$

for a dumbbell of two touching spheres, a dumbbell with $\alpha=\pi / 2$, a disk, and an ellipsoidal needle with $D_{\|} \gg D_{\perp}$. For the dumbbell-lens and ellipsoid families the modulus of the negative ratio $b_{1} \sqrt{B_{\epsilon}} / b_{2}$ is monotonically decreasing on increasing $\alpha$ from 0 to $2 \pi$ and $D_{\perp} / D_{\|}$ from 0 to $\infty$, respectively, i.e. on changing from more prolate to more oblate shapes.

\section{INDUCED INTERACTIONS}

The expressions (2.3) for the free energy cost $F$ and (2.4) for the Boltzmann factor $\exp (-\delta \mathcal{H})$ of a small particle determine the polymer-induced orientation-dependent interactions. In particular, the leading anisotropic interactions between a particle and a wall,

$$
\begin{array}{r}
F_{\text {aniso }}^{(\mathrm{p}, \mathrm{w})}=p_{0}\left(\cos ^{2} \vartheta_{\mathrm{P}}\right)\left\{b_{1} \sqrt{B_{\epsilon}} 4 \pi \sqrt{2} \mathcal{M}_{\mathrm{h}}^{\prime \prime}(y)\right. \\
\left.-b_{2}\left[f_{0}(y)+(1 / 2) f_{0}(y / 2)\right]\right\},
\end{array}
$$

with the second derivative

$$
\mathcal{M}_{\mathrm{h}}^{\prime \prime}=4\left[f_{0}(y)-(1 / 2) f_{0}(y / 2)\right]
$$


of the bulk-normalized polymer density in the half space ${ }^{\frac{5}{\underline{ }}}$ with respect to

$$
y=z_{\mathrm{P}} / \mathcal{R}_{g}, z_{\mathrm{P}}=\text { particle wall distance },
$$

and between two particles $\mathrm{P}, \mathrm{Q}$,

$$
\begin{aligned}
F_{\text {aniso }}^{(\mathrm{P}, \mathrm{Q})}= & -p_{0}\left(\cos ^{2} \varphi_{\mathrm{P}}+\cos ^{2} \varphi_{\mathrm{Q}}\right) \sqrt{2} a_{1} \sqrt{B_{\epsilon}} \beta_{\mathrm{VII}} r_{\mathrm{PQ}}^{-1} \\
& \times\left\{12 x^{-2} f_{2}(x / 2)+6 x^{-1} f_{1}(x / 2)+f_{0}(x / 2)\right\},
\end{aligned}
$$

with

$$
\begin{aligned}
& x=r_{\mathrm{PQ}} / \mathcal{R}_{g}, \\
& r_{\mathrm{PQ}}=\left|\mathbf{r}_{\mathrm{P}}-\mathbf{r}_{\mathrm{Q}}\right|=\text { particle particle distance }
\end{aligned}
$$

and

$$
\beta_{\mathrm{VII}}=\left[16 \sqrt{2} \pi b_{1} \sqrt{B_{\epsilon}}+b_{2}\right] / 2,
$$

follow from (2.3) with the half space perturbed by $\exp (-\delta \mathcal{H}) \propto \sigma_{A}(\mathrm{P})$ and the bulk perturbed by $\exp (-\delta \mathcal{H}) \propto \sigma_{I}(\mathrm{P}) \sigma_{A}(\mathrm{Q})+\sigma_{I}(\mathrm{Q}) \sigma_{A}(\mathrm{P})$, respectively. Here $f_{n}=\mathrm{i}^{n} \operatorname{erfc}$ is the n-fold iterated complementary error function, $\vartheta_{\mathrm{P}}$ is the angle between the particle axis and the surface normal of the boundary wall, and $\varphi_{\mathrm{P}}, \varphi_{\mathrm{Q}}$ are the angles between the axes of particles $\mathrm{P}, \mathrm{Q}$ and the distance vector $\mathbf{r}_{\mathrm{P}}-\mathbf{r}_{\mathrm{Q}}$ of the two particles. $F_{\text {aniso }}^{(\mathrm{P}, \mathrm{Q})}$ is proportional to the anisotropic part of $\left(\partial_{\| \mathrm{P}}^{2}+\partial_{\| \mathrm{Q}}^{2}\right) K\left(r_{\mathrm{PQ}}\right)$, with $K$ the density-density correlation function of ideal polymers in bulk solution.

For a dumbbell or lens with any $\alpha$ the particle-wall expression predicts that, for small $y$, the particle orientation parallel to the wall and, for large $y$, the perpendicular orientation, have the lowest free energies. Note that $\mathcal{M}_{\mathrm{h}}$ has a point of inflection, and $\mathcal{M}_{\mathrm{h}}^{\prime \prime}$ is positive and negative for small and large $y$, respectively. With the values $b_{1}$ and $b_{2}$ from Figures 4 and 5 above, the $b_{1}$ and $b_{2}$ contributions both favor the same, parallel orientation for small $y$. For large $y$ they favor different orientations, and their sum is $\propto-\beta_{\mathrm{VII}} f_{0}(y / 2)$, with $\beta_{\mathrm{VII}}$ from (6.7) in which $b_{1}$ dominates.

The two-particle expression predicts that particles align parallel to their distance vector, as expected from the attractive nature of the depletion interaction in a dilute polymer solution.

Qualitatively similar behavior applies for prolate and oblate ellipsoids, where $b_{1}$ and $b_{2}$ can be taken from Refs. 4, 5,11 . 


\section{SUMMARY AND CONCLUDING REMARKS}

We have studied the interaction between long flexible nonadsorbing polymers and mesoscopic colloidal dumbbells and lenses. The shape of the colloids is characterized by a parameter $\alpha$, as shown in Fig. 1 and ranges from two touching spheres for $\alpha=0$, to a sphere for $\alpha=\pi$, and to a disk for $\alpha=2 \pi$.

For small colloids and ideal polymers the amplitudes $a_{1}, b_{1}$, and $b_{2}$ in the small particle expansion (2.4), which determine the isotropic and anisotropic features of the interaction, are evaluated exactly for arbitrary $\alpha$. They follow via the general relations (3.4), (3.5), and (3.15) from the results (4.17), (4.18), and (D15) for density profiles of the Gaussian model in a wedge with opening angle $\alpha$ and Dirichlet boundary conditions and are shown as crosses in Figs. 3-5. We compare with corresponding amplitudes for ellipsoids that circumscribe and touch the dumbbells and lenses, see Eqs. (4.19), (4.20). Their values are shown as circles in Figs. 3-5. Analytical results for some special values of $\alpha$ are collected in Table I.

We also consider weakly anisotropic particles of general shapes with rotation axis and reflection center, see Eqs. (5.6)-(5.8). We find that the ratio $b_{1} / b_{2}$ in Eqs. (5.7) and (5.11) of the two anisotropy-amplitudes of these particles is independent of their shape. In particular we consider in Eqs. (5.9) the shapes of a self-intersecting torus which resembles an apple and of an american football.

How to obtain from the amplitudes $a_{1}, b_{1}$, and $b_{2}$ the orientation-dependent polymerinduced interaction between particles is discussed in Sec. VI. While the preferential alignment of two identical small particles is always parallel to their distance vector, see Eq. (6.4), the alignment of a particle with respect to a wall changes from perpendicular to parallel on decreasing the particle-wall distance, see Eq. (6.1). It would be interesting to check our predictions with simulations or real experiments.

The simple and general forms (3.6) and (3.8) of the density of the conformal stress tensor in a wedge and outside a dumbbell or lens, with eigenvectors shown in Fig. 2, follow from combining symmetries of the two geometries, see the end of Sec. III. We show in Appendix $\mathrm{C}$ that the sum (4.2) of canonical tensor and improvement term is a conformal tensor, while the separate terms are not and have densities with a more complicated form as discussed in Appendix B. 


\section{Acknowledgments}

We thank T.W. Burkhardt for useful discussions.

\section{APPENDIX A: CONTINUITY EQUATION IN THE WEDGE}

Here we show that the $\Omega$-independence of the prefactor $\tau_{i, j}$ in (3.6) follows from the continuity equation. For convenience we choose Cartesian axes perpendicular to the edge in the $\Omega=0$ and $\Omega=\pi / 2$ half planes and denote them by indices $v$ and $w$, respectively, so that

$$
\begin{aligned}
& \left(\hat{r}_{v}, \hat{r}_{w}\right)=\rho(\cos \Omega, \sin \Omega), \\
& \left(u_{v}^{(n)}, u_{w}^{(n)}\right)=(-\sin \Omega, \cos \Omega),
\end{aligned}
$$

and

$$
\left(\begin{array}{ccc}
\rho \partial \Omega / \partial \hat{r}_{v} & , \rho \partial \Omega / \partial \hat{r}_{w} \\
\partial \rho / \partial \hat{r}_{v} & , & \partial \rho / \partial \hat{r}_{w}
\end{array}\right)=\left(\begin{array}{cc}
-\sin \Omega & , \cos \Omega \\
\cos \Omega & , \sin \Omega
\end{array}\right) .
$$

Substituting (3.6) with $\tau_{i, j}(\alpha) \rightarrow \tau_{i, j}(\alpha, \Omega)$ into the two continuity equations 12

$$
\begin{aligned}
& \partial\left\langle T_{v v}\right\rangle_{\text {wedge }} / \partial \hat{r}_{v}+\partial\left\langle T_{v w}\right\rangle_{\text {wedge }} / \partial \hat{r}_{w}=0 \\
& \partial\left\langle T_{w v}\right\rangle_{\text {wedge }} / \partial \hat{r}_{v}+\partial\left\langle T_{w w}\right\rangle_{\text {wedge }} / \partial \hat{r}_{w}=0
\end{aligned}
$$

using (A1), and calculating the derivatives by means of the chain rule and (A2), one finds that the $\rho$-derivatives of the prefactor $\rho^{-d}$ cancel the $\Omega$-derivatives of the eigenvector $\mathbf{u}^{(n)}$. Only the $\Omega$-derivatives of $\tau$ remain, and the left hand sides of the first and second Eq. (A3) are given by $(\partial \tau / \partial \Omega)(d-1) / \rho^{d+1}$ multiplied by $\sin \Omega$ and $-\cos \Omega$, respectively. Thus each of the two equations implies that $\tau$ is independent of $\Omega$.

\section{APPENDIX B: WEDGE WITH $\alpha=\pi / 2$}

The propagator $\left\langle\hat{\varphi}_{12}\right\rangle_{\text {wedge }}$ for the Gaussian model in a wedge with Dirichlet boundary conditions and $\alpha=\pi / g$, with $g$ an integer, can be obtained by the method of images. Besides the half space $(\alpha=\pi)$ we consider here the simplest case of a wedge with $\alpha=\pi / 2$ in which there are three images. With the notation

$$
\hat{r}_{x i}=X_{i}, \hat{r}_{y i}=Y_{i}
$$


for the components of

$$
\hat{\mathbf{r}}_{i}=\left(\hat{\mathbf{r}}_{\mathrm{e} i}, \hat{r}_{x i}, \hat{r}_{y i}\right) \quad ; \quad i=1,2
$$

perpendicular to the edge along Cartesian axes in the two boundary half planes $\Omega=-\pi / 4$ (i.e. $Y=0, X \geq 0$ ) and $\Omega=\pi / 4$ (i.e. $X=0, Y \geq 0$ ), the propagator at the critical point has the form (4.5) with

$$
\left\langle\hat{\varphi}_{12}\right\rangle_{\mathrm{bulk}}=\tilde{S}_{d}\left[\mathbf{e}_{12}^{2}+\left(X_{1}-X_{2}\right)^{2}+\left(Y_{1}-Y_{2}\right)^{2}\right]^{-(d-2) / 2},
$$

and

$$
\begin{aligned}
\delta\left\langle\hat{\varphi}_{12}\right\rangle_{\text {wedge }}=\tilde{S}_{d}\{ & -\left[\mathbf{e}_{12}^{2}+\left(X_{1}+X_{2}\right)^{2}+\left(Y_{1}-Y_{2}\right)^{2}\right]^{-(d-2) / 2} \\
& +\left[\mathbf{e}_{12}^{2}+\left(X_{1}+X_{2}\right)^{2}+\left(Y_{1}+Y_{2}\right)^{2}\right]^{-(d-2) / 2} \\
& \left.-\left[\mathbf{e}_{12}^{2}+\left(X_{1}-X_{2}\right)^{2}+\left(Y_{1}+Y_{2}\right)^{2}\right]^{-(d-2) / 2}\right\} .
\end{aligned}
$$

Here $\mathbf{e}_{12}^{2} \equiv\left(\hat{\mathbf{r}}_{\mathrm{e} 1}-\hat{\mathbf{r}}_{\mathrm{e} 2}\right)^{2}$, and $\tilde{S}_{d}$ is defined in (4.1).

Letting $\hat{\mathbf{r}}_{1} \rightarrow \hat{\mathbf{r}}, \hat{\mathbf{r}}_{2} \rightarrow \hat{\mathbf{r}}$ on the right hand side of (B4) leads to the boundary-induced profile

$$
-\overline{\mathcal{E}}(\rho / 2)^{2-d} \sqrt{2} \equiv\left\langle\Phi^{2}(\hat{\mathbf{r}})\right\rangle_{\text {wedge }} 2^{d-2} / \tilde{S}_{d}=-X^{2-d}-Y^{2-d}+\rho^{2-d}
$$

of the energy density in (4.1), which due to

$$
X \equiv \hat{r}_{x}=\rho(\cos \Omega-\sin \Omega) / \sqrt{2}, Y \equiv \hat{r}_{y}=\rho(\cos \Omega+\sin \Omega) / \sqrt{2}
$$

is in accordance with the general form (3.1) of a scalar density with bulk exponent $x_{\mathcal{O}}=d-2$. It diverges on approaching the boundary planes $\Omega= \pm \pi / 4$.

For the boundary-induced densities of the canonical stress tensor (4.3) and the 'improvement'-term (4.4),

$$
\left(\left\langle T_{\kappa \lambda}^{(\text {can })}(\hat{\mathbf{r}})\right\rangle_{\text {wedge }},\left\langle-I_{\kappa \lambda}(\hat{\mathbf{r}})\right\rangle_{\text {wedge }}\right)=(d-2) \tilde{S}_{d} 2^{-d}\left(\mathcal{T}_{\kappa \lambda},-\mathcal{J}_{\kappa \lambda}\right)
$$

Eq. (B4) yields

$$
\begin{aligned}
& \mathcal{T}_{x x}=(d-2) Y^{-d}+d \rho^{-d-2} X^{2}-(d-1) \rho^{-d} \\
& \mathcal{T}_{x y}=d \rho^{-d-2} X Y \\
& \mathcal{T}_{\mathrm{e} \beta \mathrm{e} \beta}=(d-2)\left(X^{-d}+Y^{-d}\right)-(d-3) \rho^{-d}
\end{aligned}
$$


and

$$
\begin{aligned}
& -\mathcal{J}_{x x}=-(d-2) Y^{-d}-[d(d-2) /(d-1)] \rho^{-d-2} X^{2}+(d-2) \rho^{-d} \\
& -\mathcal{J}_{x y}=-[d(d-2) /(d-1)] \rho^{-d-2} X Y \\
& -\mathcal{J}_{\mathrm{e} \beta \mathrm{e} \beta}=-(d-2)\left(X^{-d}+Y^{-d}\right)+\left[(d-2)^{2} /(d-1)\right] \rho^{-d} .
\end{aligned}
$$

Here e $\beta$ with $\beta=3, . ., d$ runs over the $d-2$ Cartesian directions of the edge-subspace. $\mathcal{T}_{y y}$ and $-\mathcal{J}_{y y}$ follow from the above expressions for $\mathcal{T}_{x x}$ and $-\mathcal{J}_{x x}$ on exchanging $X$ and $Y$. All other components of $\left\langle T^{(\mathrm{can})}\right\rangle$ and $\langle-I\rangle$ vanish by symmetry.

In the sum $\mathcal{T}_{\kappa \lambda}-\mathcal{J}_{\kappa \lambda}$ all contributions proportional to $X^{-d}$ and $Y^{-d}$ cancel, and the density of the stress tensor $T$ in (4.2) has the simple form of (3.6) with

$$
\tau(\alpha=\pi / 2)=2^{-d} \frac{d-2}{d-1} \tilde{S}_{d}
$$

implying

$$
b_{2} / L^{d}=-2(\pi / 8)^{d / 2} / \Gamma(d / 2)
$$

if one uses (3.15) with $\mathcal{D} / L=1 / \sqrt{2}$ and the forms of $B_{T}$ below (4.4) and $\tilde{S}_{d}$ in (4.1).

However, the densities of $T^{\text {(can) }}$ and $I$ separately have nonvanishing traces, with

$$
\begin{aligned}
\mathcal{T}_{x x}+\mathcal{T}_{y y}+\sum_{\beta=3}^{d} \mathcal{T}_{\mathrm{e} \beta \mathrm{e} \beta} & =-\left\langle(\nabla \Phi)^{2}\right\rangle 2^{d-1} / \tilde{S}_{d} \\
& =(d-2)\left[(d-1)\left(X^{-d}+Y^{-d}\right)-(d-2) \rho^{-d}\right]
\end{aligned}
$$

and a more complicated form. For example, their diagonal components parallel to the edge, and the trace (B12), depend not only on $\rho$ but, due to the terms $\propto X^{-d}+Y^{-d}$, also on $\Omega$, and diverge on approaching the boundaries $X=0$ or $Y=0$. There is a corresponding divergence of the diagonal components of $T^{(\mathrm{can})}$ and $I$ parallel to a Dirichlet boundary wall for the half space $(\alpha=\pi)$. Moreover, $\mathbf{u}^{(n)}$ of Eq. (3.6) and Fig. 2 is, in general, not

an eigenvector of $\left\langle T^{(\mathrm{can})}\right\rangle$, due to the terms proportional to $Y^{-d}$ and $X^{-d}$ in $\mathcal{T}_{x x}$ and $\mathcal{T}_{y y}$, respectively. In the continuity equation satisfied by $\left\langle T^{(\mathrm{can})}\right\rangle$, these terms do not contribute.

\section{APPENDIX C: CONFORMAL STRESS DENSITY}

Here we verify that in the Gaussian model the boundary-induced profile $\langle T\rangle$, with $T$ the sum (4.2) of the canonical stress tensor and the improvement term, is a conformal tensor. 
For a conformal transformation $\hat{\mathbf{r}}^{\prime} \rightarrow \mathbf{r}^{\prime}$ which leads from a geometry $\hat{G}^{\prime}$ to a geometry $G^{\prime}$, we use the transformation law

$$
\delta\left\langle\Phi\left(\mathbf{r}_{1}^{\prime}\right) \Phi\left(\mathbf{r}_{2}^{\prime}\right)\right\rangle_{G^{\prime}}=\left(b\left(\hat{\mathbf{r}}_{1}^{\prime}\right) b\left(\hat{\mathbf{r}}_{2}^{\prime}\right)\right)^{(d-2) / 2} \delta\left\langle\Phi\left(\hat{\mathbf{r}}_{1}^{\prime}\right) \Phi\left(\hat{\mathbf{r}}_{2}^{\prime}\right)\right\rangle_{\hat{G}^{\prime}}
$$

for the propagator $\delta\langle\Phi \Phi\rangle$ with bulk contribution subtracted as in (4.5). The dilatation factor $b\left(\hat{\mathbf{r}}^{\prime}\right)$ of the transformation is defined below Eq. (3.9). Consider the boundaryinduced density $\left\langle T_{k l}\left(\mathbf{r}^{\prime}\right)\right\rangle_{G^{\prime}}$, where $T_{k l}$ follows from (4.2) on replacing $(\kappa, \lambda, \hat{\mathbf{r}})$ by $\left(k, l, \mathbf{r}^{\prime}\right)$. As in Appendix B each of its contributions follows from the subtracted propagator on the left hand side of ([C1) by appropriate differentiations, where for the terms in $-\left\langle I_{k l}\right\rangle_{G^{\prime}}$ and $\left\langle T_{k l}^{(\text {can })}\right\rangle_{G^{\prime}}$ the arguments $\mathbf{r}_{1}^{\prime}$ and $\mathbf{r}_{2}^{\prime}$ are set equal to $\mathbf{r}^{\prime}$ before and after differentiating, respectively. Following the same steps on the right hand side of ([C1), one finds that in the sum $\left\langle T_{k l}\right\rangle_{G^{\prime}}=\left\langle T_{k l}^{(\mathrm{can})}\right\rangle_{G^{\prime}}-\left\langle I_{k l}\right\rangle_{G^{\prime}}$ the contributions involving $\mathbf{r}^{\prime}$-derivatives of $b$ that come from calculating $T^{(\text {can) }}$ cancel the contributions involving $\mathbf{r}^{\prime}$-derivatives of $\partial \hat{\mathbf{r}}^{\prime} / \partial \mathbf{r}^{\prime}$ and $b$ that come from calculating $-I$ and one is left with the transformation formula

$$
\left\langle T_{k l}\left(\mathbf{r}^{\prime}\right)\right\rangle_{G^{\prime}}=\sum_{\kappa, \lambda} b^{d-2}\left(\hat{\mathbf{r}}^{\prime}\right)\left(\frac{\partial \hat{r}_{\kappa}^{\prime}}{\partial r_{k}^{\prime}}\right)\left(\frac{\partial \hat{r}_{\lambda}^{\prime}}{\partial r_{l}^{\prime}}\right)\left\langle T_{\kappa \lambda}\left(\hat{\mathbf{r}}^{\prime}\right)\right\rangle_{\hat{G}^{\prime}}
$$

of a conformal tensor. In general $T^{\text {(can) }}$ and $I$ separately do not satisfy a transformation law of the form of (C2). It is instructive to explicitly check the above arguments for the inversion $\hat{\mathbf{r}}^{\prime}=L^{2} \mathbf{r}^{\prime} / r^{\prime 2}$, where

$$
\frac{\partial \hat{r}_{\kappa}^{\prime}}{\partial r_{k}^{\prime}}=b\left(\hat{\mathbf{r}}^{\prime}\right) \mathcal{I}_{\kappa, k}\left(\mathbf{r}^{\prime}\right) \quad, \quad b\left(\hat{\mathbf{r}}^{\prime}\right)=\frac{L^{2}}{r^{\prime 2}},
$$

with $\mathcal{I}$ defined in Eq. (3.12), and where (C2) reduces to the transformation law of Eqs. (2.36) and (2.37) in Ref. 9 .

The transformation formula (C2) for the stress tensor density is also expected to apply beyond the Gaussian model. Given in geometry $\hat{G}^{\prime}$ an eigenvector of $\left\langle T\left(\hat{\mathbf{r}}^{\prime}\right)\right\rangle_{\hat{G}^{\prime}}$ which points along a certain distance vector $d \hat{\mathbf{r}}^{\prime}$ and belongs to an eigenvalue $\hat{t}$, Eq. (C2) implies in geometry $G^{\prime}$ an eigenvector of $\left\langle T\left(\mathbf{r}^{\prime}\right)\right\rangle_{G^{\prime}}$ which points along the corresponding distance vector $d \mathbf{r}^{\prime}$ and belongs to the eigenvalue $b^{d}\left(\hat{\mathbf{r}}^{\prime}\right) \hat{t}$. 


\section{APPENDIX D: STRESS AMPLITUDE FOR ARBITRARY OPENING ANGLE}

It is convenient to calculate $\tau(\alpha)$ from (3.6) with $\kappa=\lambda$ equal to an edge direction e $\beta$. Using (4.2)-(4.4) and the property

$$
2\left\langle(\nabla \Phi)^{2}\right\rangle_{\text {wedge }}=\Delta\left\langle\Phi^{2}\right\rangle_{\text {wedge }}
$$

of the vanishing trace of $\left\langle T^{(\mathrm{can})}-I\right\rangle_{\text {wedge }}$, this yields

$$
\tau(\alpha)=\rho^{d}\left\langle T_{\mathrm{e} \beta \mathrm{e} \beta}\right\rangle=\rho^{d}\left[\left\langle\left(\Phi_{\mathrm{e} \beta}\right)^{2}\right\rangle_{\text {wedge }}-\frac{1}{4(d-1)} \Delta\left\langle\Phi^{2}\right\rangle_{\text {wedge }}\right],
$$

where

$$
\Phi_{\mathrm{e} \beta}(\hat{\mathbf{r}})=\partial \Phi(\hat{\mathbf{r}}) / \partial \hat{r}_{\mathrm{e} \beta} .
$$

Since the boundary induced profile

$$
\left\langle\left(\Phi_{\mathrm{e} \beta}(\hat{\mathbf{r}})\right)^{2}\right\rangle_{\text {wedge }}=\lim _{\hat{\mathbf{r}}_{1} \rightarrow \hat{\mathbf{r}}, \hat{\mathbf{r}}_{2} \rightarrow \hat{\mathbf{r}}}\left[\left\langle\Phi_{\mathrm{e} \beta}\left(\hat{\mathbf{r}}_{1}\right) \Phi_{\mathrm{e} \beta}\left(\hat{\mathbf{r}}_{2}\right)\right\rangle_{\text {wedge }}-\left\langle\Phi_{\mathrm{e} \beta}\left(\hat{\mathbf{r}}_{1}\right) \Phi_{\mathrm{e} \beta}\left(\hat{\mathbf{r}}_{2}\right)\right\rangle_{\text {bulk }}\right]
$$

is, apart from a factor $\tilde{S}_{d}$, analytic ${ }^{21}$ in $d$, the calculation proceeds similar to (4.11)-(4.17): We consider $d<0$, where

$$
\left\langle\Phi_{\mathrm{e} \beta}\left(\hat{\mathbf{r}}_{1}\right) \Phi_{\mathrm{e} \beta}\left(\hat{\mathbf{r}}_{2}\right)\right\rangle_{\mathrm{bulk}}=(d-2)\left[\left|\hat{\mathbf{r}}_{1}-\hat{\mathbf{r}}_{2}\right|^{2}-d\left(\hat{r}_{1 \mathrm{e} \beta}-\hat{r}_{2 \mathrm{e} \beta}\right)^{2}\right]\left|\hat{\mathbf{r}}_{1}-\hat{\mathbf{r}}_{2}\right|^{-2-d} \tilde{S}_{d}
$$

does not contribute on the right hand side of (ID4), and find for $\Omega=0$

$$
\left.\rho^{d}\left\langle\left(\Phi_{\mathrm{e} \beta}(\hat{\mathbf{r}})\right)^{2}\right\rangle_{\text {wedge }}\right|_{\Omega=0}=2 \pi^{(2-d) / 2} \frac{1}{\alpha \Gamma(1-(d / 2))} J_{\mathrm{e}},
$$

with

$$
J_{\mathrm{e}}=\int_{0}^{1} d t \Xi_{d}(t) t^{\pi / \alpha} \frac{1}{1-t^{2 \pi / \alpha}}
$$

where

$$
\Xi_{d}(t)=t^{(d-2) / 2}(1-t)^{-d} .
$$

Here we have rewritten $\left\langle\left(\Phi_{\mathrm{e} \beta}\right)^{2}\right\rangle_{\text {wedge }}$ as $\sum_{\beta}\left\langle\left(\Phi_{\mathrm{e} \beta}\right)^{2}\right\rangle_{\text {wedge }} /(d-2)$ and used Eqs. (4.7) and (4.8) with $2 A=d$. The integral (D7) converges for $-2 \pi / \alpha<d<0$.

Writing $J_{\mathrm{e}}$ as the sum of

$$
J_{\mathrm{e}}^{(1)}=\int_{0}^{1} d t \Xi_{d}(t) t^{\pi / \alpha}\left[\frac{1}{1-t^{2 \pi / \alpha}}-k(t)\right]
$$

and

$$
J_{\mathrm{e}}^{(2)}=\int_{0}^{1} d t \Xi_{d}(t) t^{\pi / \alpha} k(t),
$$


with

$$
k(t)=\frac{\alpha}{2 \pi} \frac{1}{1-t}+\frac{1}{2}\left(1-\frac{\alpha}{2 \pi}\right)+\frac{1}{24}\left(\frac{2 \pi}{\alpha}-\frac{\alpha}{2 \pi}\right)\left[2(1-t)+(1-t)^{2}\right],
$$

the continuation to $d=3$ leads to

$$
J_{\mathrm{e}}^{(2)}(\alpha, 3)=\frac{1}{72}\left[\left(\frac{2 \pi}{\alpha}\right)^{2}-1+6 \frac{\alpha}{2 \pi}\right]
$$

yielding

$$
\left.\left\langle\left(\Phi_{\mathrm{e} \beta}(\hat{\mathbf{r}})\right)^{2}\right\rangle_{\text {wedge }}\right|_{\Omega=0} \rho^{d}=-\frac{1}{\alpha \pi}\left[J_{\mathrm{e}}^{(1)}(\alpha, 3)+J_{\mathrm{e}}^{(2)}(\alpha, 3)\right]
$$

in $d=3$ where $J_{\mathrm{e}}^{(1)}(\alpha, 3)$ follows from ( $(\underline{\mathrm{D} 9})$ on replacing $\Xi_{d}$ by $\Xi_{3}=t^{1 / 2}(1-t)^{-3}$.

In the second contribution to $\tau$ in (D2),

$$
\rho^{d} \Delta\left\langle\Phi^{2}\right\rangle_{\text {wedge }}=-\sqrt{2} \tilde{S}_{d}\left\{\partial_{\Omega}^{2} \overline{\mathcal{E}}+(d-2)^{2} \overline{\mathcal{E}}\right\}
$$

can, for $\Omega=0$ and $d=3$, be expressed by $e_{0}$ and $e_{2}$ in (4.17), (4.18), so that

$$
\tau(\alpha)=-\frac{1}{\alpha \pi}\left[J_{\mathrm{e}}^{(1)}(\alpha, 3)+J_{\mathrm{e}}^{(2)}(\alpha, 3)\right]+\frac{1}{16 \sqrt{2} \pi} e_{0}(\alpha)\left[e_{2}(\alpha)+1\right] .
$$


1 D. Rudhardt and C. Bechinger and P. Leiderer, Phys. Rev. Lett. 81, 1330 (1998).

2 A. van Blaaderen, Science 301, 470 (2003); MRS Bulletin, February, 85 (2004).

3 E. Snoeks et al. Adv. Mater. 12, 1511 (2000).

4 E. Eisenriegler, A. Bringer, and R. Maaßen. J. Chem. Phys. 118, 8093 (2003).

5 E. Eisenriegler, in Soft Condensed Matter, (G. Gompper and M. Schick eds.), Wiley-VCH (2005).

6 P.G. de Gennes, Scaling Concepts in Polymer Physics (Cornell University, Ithaca, 1979).

7 T. Burkhardt and E. Eisenriegler, Phys. Rev. Lett. 74, 3189 (1995); 78, 2867 (1997).

8 E. Eisenriegler and U. Ritschel, Phys. Rev. B51, 13717 (1995).

9 E. Eisenriegler, J. Chem. Phys. 121, 3299 (2004).

10 First, Eq. (2.4) applies to correlation functions with operators much further away from $\mathbf{r}_{\mathrm{P}}$ than the particle size. Apart from contact-terms ${ }^{4}$, Eq. (2.4) may also be used for calculating integrals such as (2.3). Here we only consider the leading isotropic and anisotropic contributions for small particle size, and the contact terms do not contribute. The leading contact term has particle size exponent $d$ and is isotropic $\stackrel{4}{ }$.

11 Compare the discussion in Refs. 4, 5 for the Gaussian model. In the notation of Ref. 5 with amplitudes $\beta$ in Eqs. (162-165), $a_{1} \sqrt{B_{\epsilon}}=\beta_{\mathrm{I}} \tilde{S}_{d} / \sqrt{2}, b_{1} \sqrt{B_{\epsilon}}=\left(\beta_{\mathrm{VI}}(d-2)+\beta_{\mathrm{VII}} d\right) \tilde{S}_{d} /(4 \sqrt{2}(d-$ $1)), b_{2}=\left(-\beta_{\mathrm{VI}}+\beta_{\mathrm{VII}}\right) / 2$ with $\tilde{S}_{d}$ given in (4.1).

12 J.L. Cardy in Phase Transitions and Critical Phenomena, edited by C. Domb and J.L. Lebowitz (Academic Press, London, 1986), Vol. 11, p. 55.

13 T. Burkhardt and E. Eisenriegler, J. Physics A 18, L83 (1985).

14 A. Hanke, M. Krech, F. Schlesener. and S. Dietrich, Phys. Rev. E 60, 5163 (1999).

15 While we concentrate in Secs. IV-VI on nonadsorbing ideal chains represented by the Gaussian model with Dirichlet boundary conditions, the discussion in Sec. III does not specify the bulk and surface universality classes $\frac{12}{2}$ and allows also for other applications 9.14 .

16 J.L. Cardy, Nucl. Phys. B290, 355 (1987).

17 D.M. Mc Avity and H. Osborn, Nucl. Phys. B455, 522 (1995).

18 (a) T.W. Burkhardt and T. Xue, Phys. Rev. Lett. 66, 895 (1991); (b) Nucl. Phys. B354, 653 (1991); (c) T.W. Burkhardt and I. Guim, Phys. Rev. B36 2080 (1987); (d) Phys. Rev. B47 14 
306 (1993).

19 (a) L.S. Brown, Ann. Phys. (N.Y.) 126, 135 (1980); (b) L.S. Brown and J.C. Collins, ibid. 130, 215 (1980).

20 J.L. Cardy, J. Phys. A16, 3617 (1983).

21 For $\alpha=\pi / g$, where $\delta\left\langle\hat{\varphi}_{12}\right\rangle_{\text {wedge }}$ is a superposition of images, $\overline{\mathcal{E}}$ is an entire function of $d$. For example, $\overline{\mathcal{E}}$ is given by $(2 \cos \Omega)^{2-d} / \sqrt{2}$ if $\alpha=\pi$, and by (B5) if $\alpha=\pi / 2$. For $\alpha$ arbitrary, the analytic continuation of $(\sqrt{2} / \alpha) \sin (\pi d / 2) J^{(2)}$ with $J^{(2)}$ from (4.15), and thus of $e_{0}$, are analytic functions of $d$ in the interval $2-(2 \pi / \alpha)<d<4$. The continuation of $e_{0}$, after taking the limit in (4.11) below $d=2$, coincides with the result from taking the limit above $d=2$ directly, since both certainly coincide for $\alpha=\pi / g$ and are analytic in $\alpha$. Similar statements apply to $J_{\mathrm{e}}$ in (D7). Here the continuation of $J_{\mathrm{e}} / \tilde{S}_{d}$ is an analytic function of $d$ for $-(2 \pi / \alpha)<d<4$.

22 The contributions $3 \beta_{\mathrm{I}}^{2} \Phi^{4} / 4$ ! and $-15 \beta_{\mathrm{I}}^{3} \Phi^{6} / 6$ ! of the nonleading isotropic operators $\Phi^{4}$ and $\Phi^{6}$ to $\sigma_{I}$ on the right hand side of Eq. (2.5) follow from the relations $\left\langle\Phi^{4}\right\rangle_{\text {particle }}=3\left\langle\Phi^{2}\right\rangle_{\text {particle }}^{2}$ and $\left\langle\Phi^{6}\right\rangle_{\text {particle }}=15\left\langle\Phi^{2}\right\rangle_{\text {particle }}^{3}$ between profiles due to Wick's theorem. Here $\beta_{\text {I }}$ equals $a_{1} \sqrt{B_{\epsilon}} \sqrt{2} / \tilde{S}_{d}$ and appears in the leading isotropic contribution $a_{1} \epsilon=-\beta_{\mathrm{I}} \Phi^{2} / 2$ in the notation of Ref. 5 . For the sphere with $\alpha=\pi$ and $\beta_{\mathrm{I}}=(L / 2)^{d-2} / \tilde{S}_{d}$ and the dumbbell of two touching spheres with $\alpha=0$ and $\beta_{\mathrm{I}}=(L / 2)^{d-2} 2\left(1-2^{3-d}\right) \zeta(d-2) / \tilde{S}_{d}$ the role of these nonleading operators has been discussed in Refs. 4 and 9 , respectively.

23 For the circumscribing ellipsoids in Eqs. (4.19) and (4.20), the parameters $\left[f, \xi_{\mathrm{E}}\right]$ of Ref. 5 are given by $\left[2^{-1 / 2} L \cos (\alpha / 4) \sqrt{\cos (\alpha / 2)}, 2^{1 / 2} / \sqrt{1-\operatorname{tg}^{2}(\alpha / 4)}\right]$ and $\left[(\mathcal{D} / 2) \sqrt{1-\operatorname{ctg}^{2}(\alpha / 4)}, 1 / \sqrt{\operatorname{tg}^{2}(\alpha / 4)-1}\right]$, respectively.

${ }^{24}$ For example from (5.4) one may calculate $\left\langle\Phi^{2}(\mathbf{r})\right\rangle$ and $\left\langle T_{k l}(\mathbf{r})\right\rangle$, and from the behavior for $R \ll r$ obtain $a_{1}, b_{1}$, and $b_{2}$. 


\begin{tabular}{|c|c|c|c|c|c|}
\hline$\alpha$ & 0 & $\pi / 2$ & $\pi$ & $2 \pi$ & $\alpha$ \\
\hline$a_{1} \sqrt{B_{\epsilon}} / L$ & $\begin{array}{c}2^{-1 / 2} \ln 2 \\
=0.490 \\
(0.569)\end{array}$ & $\begin{array}{c}2^{-1 / 2}-2^{-2} \\
\quad=0.457 \\
(0.508)\end{array}$ & $\begin{aligned} & 2^{-3 / 2} \\
= & 0.354\end{aligned}$ & $\begin{array}{l}2^{-1 / 2} \pi^{-1} \\
=0.225\end{array}$ & $a_{1} \sqrt{B_{\epsilon}} / \mathcal{D}$ \\
\hline$b_{1} \sqrt{B_{\epsilon}} / L^{3}$ & $\begin{array}{c}7 \zeta(3) /(64 \sqrt{2}) \\
=0.0930 \\
(0.0561)\end{array}$ & $\begin{array}{c}\left(4+2^{-1 / 2}\right) /(64 \sqrt{2}) \\
=0.0520 \\
(0.0304)\end{array}$ & 0 & $\begin{array}{c}-5 /(96 \pi \sqrt{2}) \\
=-0.0117\end{array}$ & $b_{1} \sqrt{B_{\epsilon}} / \mathcal{D}^{3}$ \\
\hline$b_{2} / L^{3}$ & $\begin{array}{l}-\pi \zeta(3) / 4 \\
=-0.944 \\
(-0.630)\end{array}$ & $\begin{array}{l}-\pi 2^{-5 / 2} \\
=-0.555 \\
(-0.346)\end{array}$ & 0 & $\begin{aligned} & 1 / 6 \\
= & 0.166\end{aligned}$ & $b_{2} / \mathcal{D}^{3}$ \\
\hline
\end{tabular}

TABLE I: Amplitudes for dumbbells with $\alpha=0, \pi / 2$, the sphere $(\alpha=\pi)$, and the disk $(\alpha=$ $2 \pi$ ) in three spatial dimensions. Numbers in brackets denote amplitudes for prolate ellipsoids circumscribing the dumbbells. The values correspond to the crosses and circles in Figs. 3-5. 

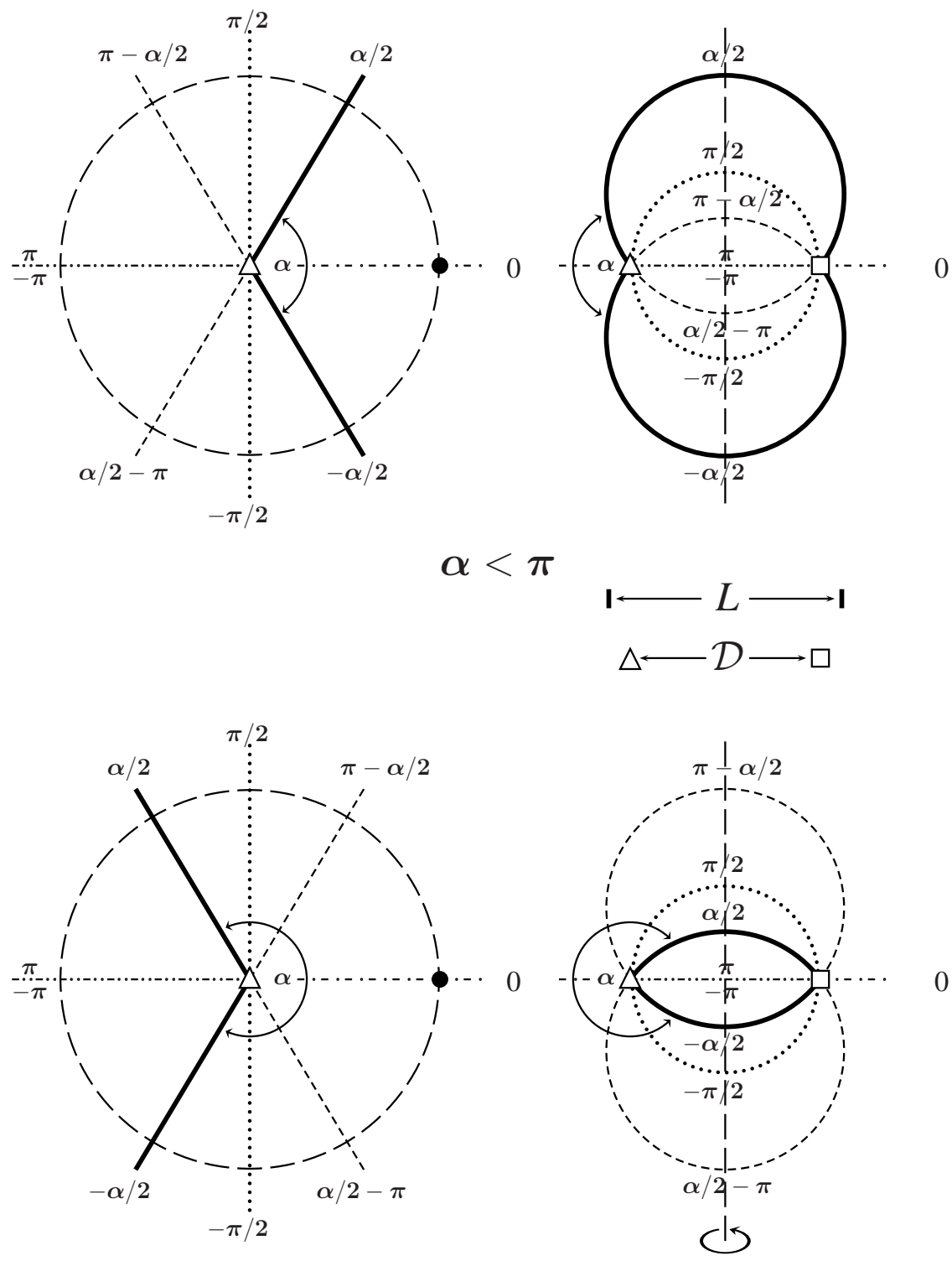

$$
\alpha>\pi
$$

FIG. 1: Conformal mapping of a wedge onto a dumbbell or lens. 

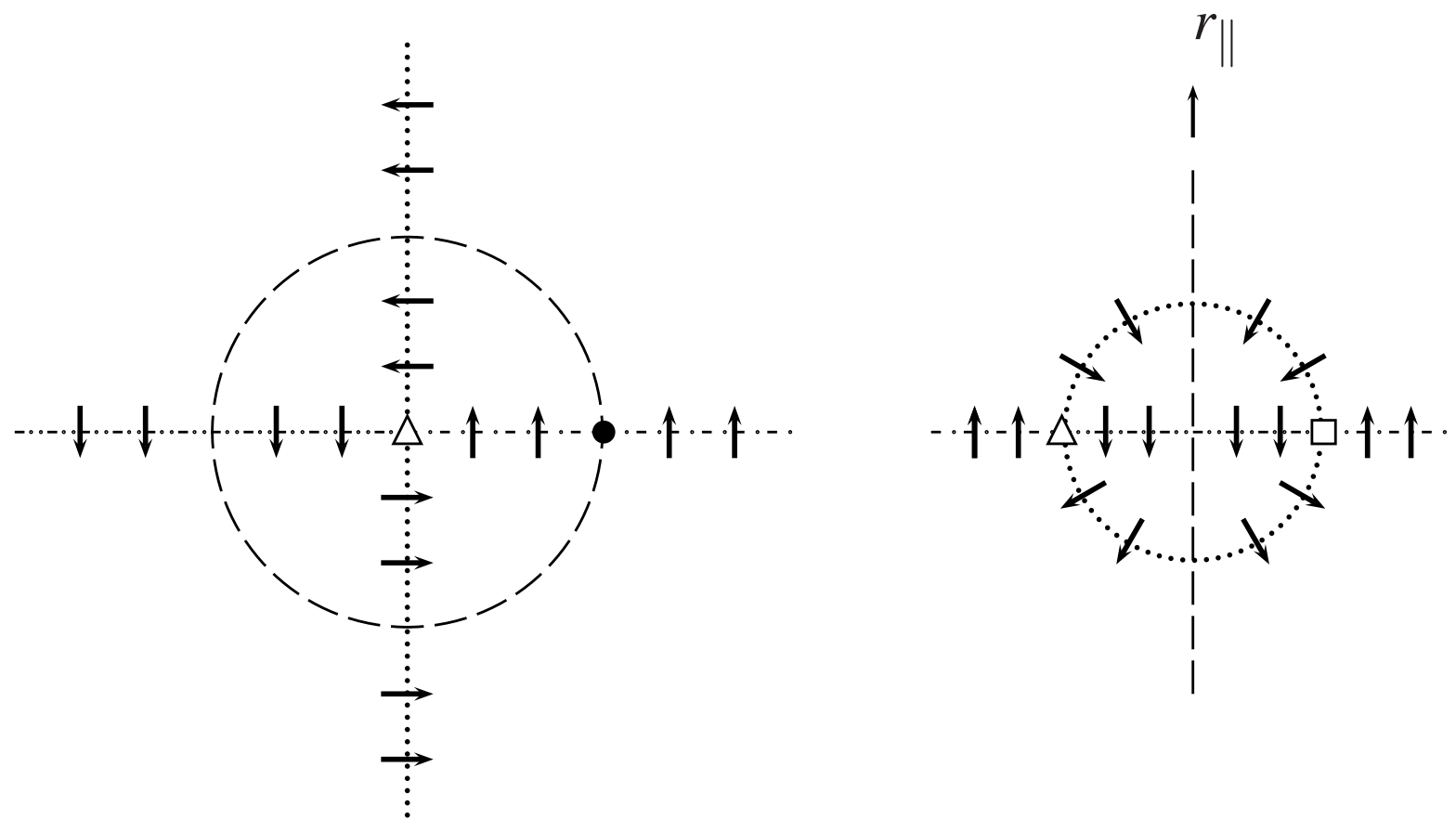

FIG. 2: The normalized eigenvectors $\mathbf{u}^{(n)}(\Omega)$ (left) and $\mathbf{u}^{(N)}(\mathbf{r})$ (right) of the stress tensor density in a wedge and outside a dumbbell or lens. The vector field $\mathbf{u}^{(N)}$ is determined by Eq. (3.10). The symbols for lines and points correspond to those in Fig. 1. 


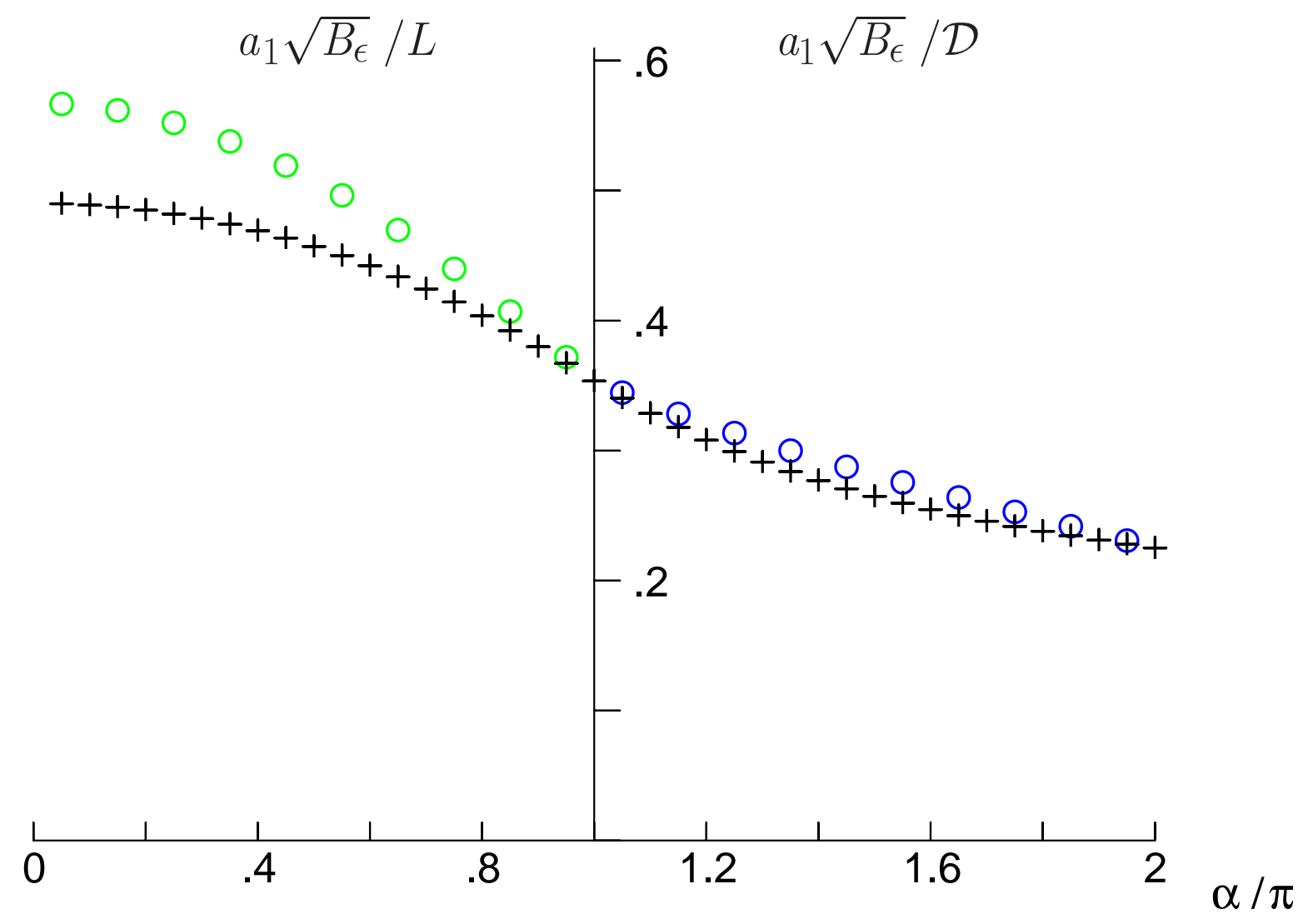

FIG. 3: The leading amplitude $a_{1}$ in (2.4) for particles with Dirichlet boundary conditions in the Gaussian model. Crosses denote results for dumbbells $(0<\alpha / \pi<1)$ and lenses $(1<\alpha / \pi<2)$. Circles denote results for circumscribing prolate and oblate ellipsoids, see the paragraph containing Eqs. (4.19) and (4.20). The oblate ellipsoid and the lens coincide for $\alpha / \pi=1$ and 2, where they become a sphere and a disk, respectively. 


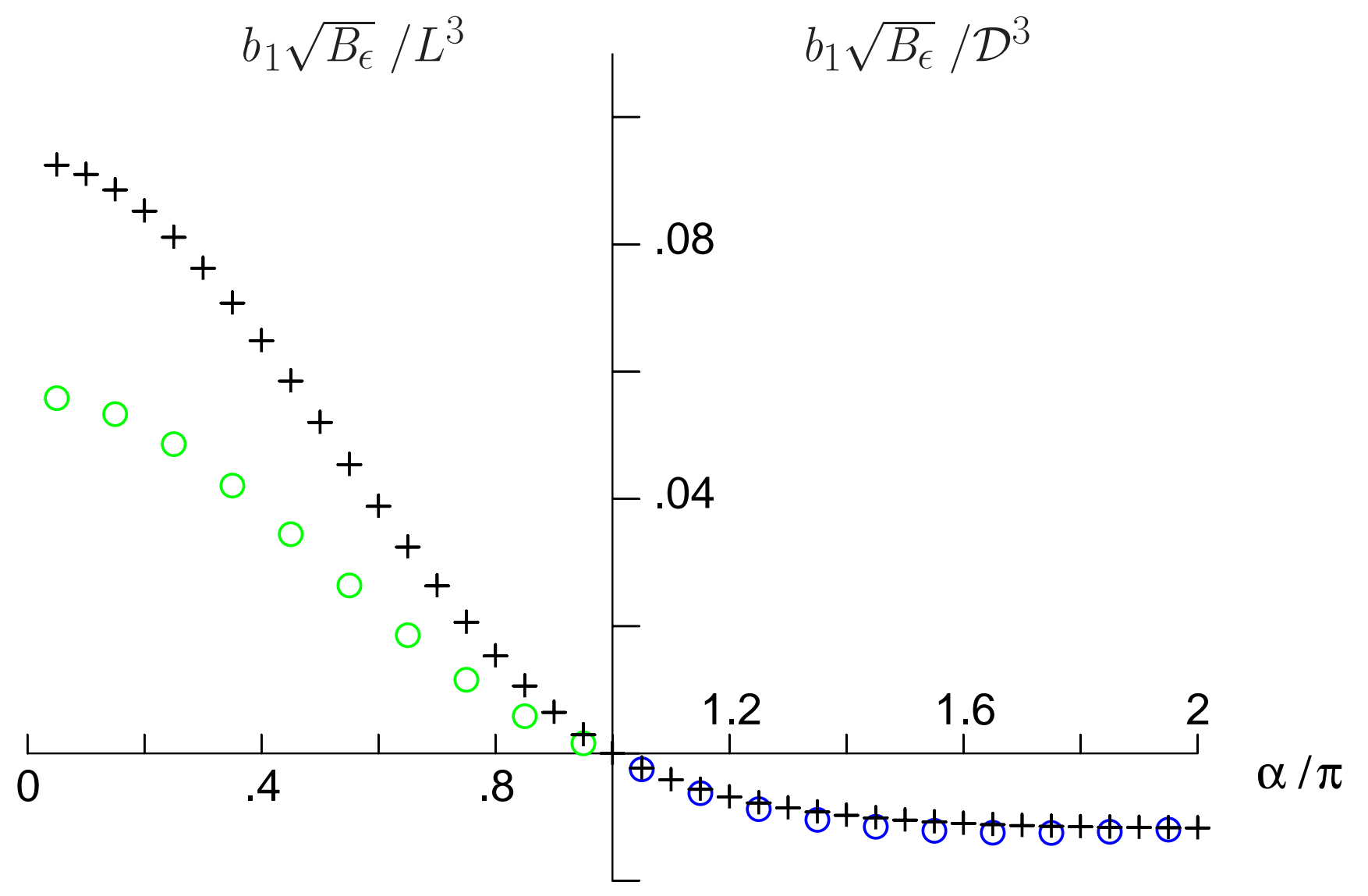

FIG. 4: The anisotropy amplitude $b_{1}$ in (2.4). 


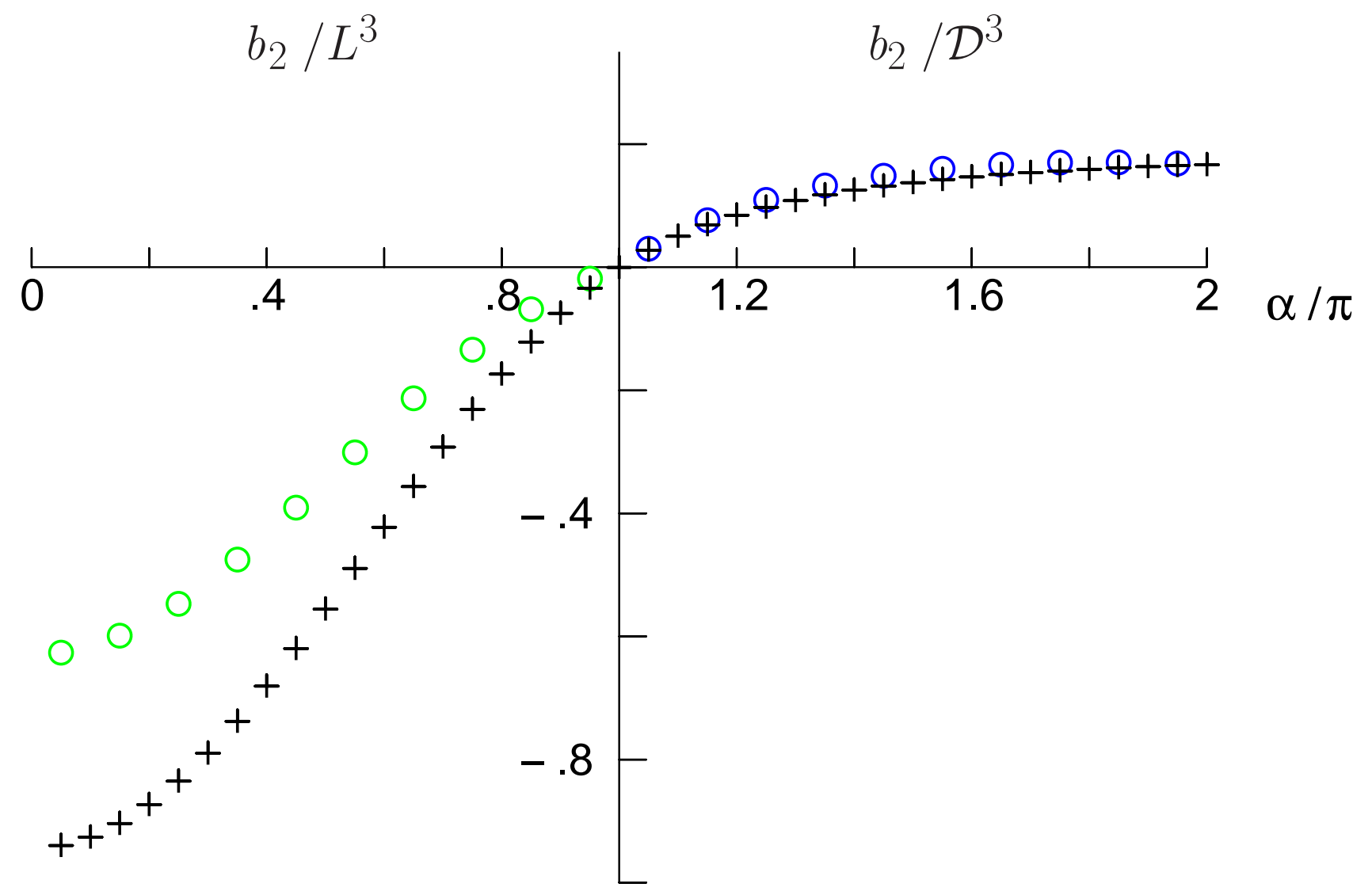

FIG. 5: The anisotropy amplitude $b_{2}$ in (2.4). 